was detected. Further aspirations produced no benefit, but an increased dose of steroids helped. However, two weeks later she developed severe respiratory failure and died. At necropsy the lungs showed prominent arterial radicles, a moderate amount of oedema, and scattered, mainly subpleural, areas suggestive of chronic pneumonitis. Both latter features were worse on the left (weight 985g.) than on the right (weight $720 \mathrm{~g}$.) Both pleural spaces contained old fibrous adhesions and bilateral yellow effusions. The heart (weight $360 \mathrm{~g}$.) showed right ventricular hypertrophy (10mm.) and dilatation; the right auricle was normal. There was no thrombosis in the syst-mic vessels.

The lungs were sent to Professor Herbert Spencer for examination. In his opinion they showed: (1) Some pulmonary arterial thickening; occasional arteries also showed recanalization; (2) interstitial fibrosis (Fig.) plus recent intra-alveolar fibrinous oedema; (3) pronounced dilatation of the bronchial veins; (4) enormous distension of the alveolar capillaries in places; and (5) thrombosis of small- and medium-sized veins with evidence of recanalization.

These changes add up to thrombosis of the pulmonary venous return with subsequent oedema, collateral channel formation, and pulmonary artery hypertension (with occasional arterial thrombosis and recanalization).

The disease was an evolving condition in this patient as is apparent from the varying degrees of oedema and venous occlusion. Despite this activity no aetiological agent was discovered. Perhaps virus culture of the sputum would have been revealing, since Liebow suggests haemagglutination associated with pulmonary viral infections is possibly the cause of the thrombosis.

More information could have been forthcoming had the patient been diagnosed in life. The object of this report is to remind clinicians and pathologists of the disease, and to underline the opinion of previous authors that it may not be as rare as the literature suggests.-We are, etc.,

\section{JAMES BRIGGS.}

\section{A. T. M. ROBERTS}

Frenchay Hospital,
Bristol.

REFERENCES Brown, C. H., and Harrison, C. V., Lancet, 1966,
2. 61.

Clinicopatholooical Conference, British Medical Fournal, 1968, 1, 818.

Liebow, A. A., McAdams, A. J.. Carrington C. B., and Viamonte, M., Circulation, 1967, 36.
suppl. No. 2, p. 172.

\section{Caring for the Elderly}

SIR,-The article "Evaluation of Early Diagnostic Services for the Elderly" (1 August, p. 275) sheds a welcome gleam of light on a hitherto almost unexplored field and raises a number of interesting points. As the authors showed, these are not symptomless patients, but patients with an illness looking for a doctor.

The current pessimism over early diagnosis and treatment of the elderly is injustified. Cannot the work carried out in the survey be done almost as well by the reneral practitioner backed by an ordinary outpatient department? I myself, handicapped by lack of staff and time, have done something along the same lines for 10 years and have obtained gratifying results.

Early diagnosis for this type of patient is just the start of a long marathon of care and maintenance which will last as long as the life of the patient-perhaps 10 or 15 \%ars which one hopes will avoid the invalid stage of life and, as a result of one's efforts, enable the patients to die with their boots on. The figure of $42 \%$ improved of those patients treated is surely too low. No mention is made of the treatments given, but one wonders if such treatments as isoxsuprine for cerebral ischaemia, verapamil and related drugs for angina, and indomethacin and iboprufen for arthritis have been used. All these treatments inay slow down or even arrest deterioration, but the results are better judged after five or even 10 years instead of a mere 18 months.

Finally, it is stated that it is unlikely that general practitioners will be able to accept the burden of this type of work, and that it would be best left to the geriatricians. Thev will not, of course, be able to do this or indeed many other types of work if any available finance which properly belongs to the primary care services is siphoned off to highly expensive and geographically remote specialist centres, which will never be able to provide the basic care which this underprivileged group of patients so desperately need.-I am, etc.,

$$
\begin{gathered}
\text { East Hoathly, } \\
\text { near Lewes. } \\
\text { Sussex. }
\end{gathered}
$$

\section{G. Elliott.}

\section{Primary Care of Patients with Skin Disease}

SIR,-It seems that the summary (30 May, p. 535) of the B.M.A. Planning Unit working party's report on Primary Medical Care has given rise to alarm and despondency and protest in some parts of the country, with particular reference to its suggestion that all patients with diseases of the skin should be seen initially at a hospital clinic.

It does not seem to have been appreciated that this is simply the opinion of the working party, and that it did not at any time consult the B.M.A. Dermatologists' Group Committee or any of its members.

I would like, therefore, to disclaim any responsibility for this opinion, and to say that this in no way represents the view taken by dermatologists or their representatives. I am, etc.,

IAN W. Caldwell, Chairman, B.M.A. Dermatologists Southampton, Hants. Group Committee.

\section{Prophylaxis of Pulmonary Embolism}

SIR,-We are concerned that in the "Current Practice" article (27 June, p. 780) on the prophylaxis of pulmonary embolism, no mention was made of the various mechanical approaches to the problem where anticoagulants have failed or are contraindicated -for example, methods such as partial occlusion of the inferior vena cava by clip, filter, or plication.

In desperately ill patients recurrent embolism may be prevented temporarily by inserting a form of umbrella into the inferior vena cava (Eichelter, Mobin-Uddin). ${ }^{12}$ If an operation is possible an easy, safe, and effective method is the application of an inferior vena caval clip that flattens the vessel to a slit of about $5 \mathrm{~mm}$., a size that will not transmit a lethal embolus. The clip method of partial inferior vena caval occlusion is easier and more effective than either the filter ${ }^{3}$ or the plication, ${ }^{4}$ and although it seems to increase the incidence of peripheral thrombophlebitis we consider that its prophylactic use is justified in patients who are at particular risk from embolism. Preliminary results of a prospective controlled trial indicate that it saves lives. One of us wears a caval clip.-We are, etc.,

\section{A. M. N. GARDNER R. L. MIDGLEY. \\ M. J. TURNER C. C. Wilmshurst.}

Torbay Hospital.
Torquay, Devon

\section{REFERENCES} Eichelter, P., and Schenk, W. G., jun., Archives
of Surferv, 1968.97 .348

Mobin-Uddin, K., Medical Post, 4 November, 1969. p. 26

De Weese, M. S., and Hunter, D. C., Archives of Surrery. 1963, 86. 852 .

Spencer, F. C., Jude, J.. Reinhoff. W. F., and
Stonesifer, G., Annals of Surrery, 1965, 16i. 788.

\section{Acute Malaria in Newborn Infants}

SIR,-Dr. N. E. Okeke's observations (11 Julv, p. 108) on the frequent occurrence of congenitally acquired malaria in Enugu (Nigeria) contrast sharply with our findings in the Gambia. At our hospital we see and treat many cases of malaria in young children each year, yet in more than 20 years one of us (I. A. McG.) cannot recollect seeing any infection which seemed likely to have been acquired in this way.

We have recently completed a study of malaria infections in parturient women and their newborn infants, the detailed results of which will be published in full later. Thick films of placental, maternal peripheral, and neonatal peripheral blood were examined for the presence of malaria parasites. The bulk of the films of peripheral blood were taken from mother and child within 24 hours of birth; a few were taken between 24 and 36 hours, and one was taken at 56 hours. A hundred microscope fields were examined before any film was considered free of parasites. Two hundred and twentyseven mothers and their 227 children comprised the study group. Seventy-four (32\%) placentae were found parasitized, some very heavily so. Parasites were found in maternal peripheral blood in $65(28 \%)$ of cases, but were not seen in any blood film made from the infants

It is probable that Gambian women diffe from Dr. Okeke's Nigerian patients in respect of the frequency with which they receive malarial infections and of the access they command to treatment facilities. One hundred and eleven of our maternal group were residents of the city of Bathurst within the precincts of which mosquito control is regularly practised and treatment facilities are readily available. These women showed a relatively low prevalence of parasitaemia in peripheral and in placental blood; the rate for each being $12 \%$. The remaining (116) women were residents in semi-uliban and rural areas of the country where mosquito control is not practised, and where treatment facilities are much less available. Placental infection was found in $48 \%$ of these women and peripheral parasitaemia in $43 \%$. 